\title{
Enamel Resistance to Demineralization and Bracket Debonding with ACTIVA $^{\mathrm{TM}}$ BioACTIVE-Cement
}

\section{Silvestrini $\mathbf{M}^{1}$, Tran $\mathrm{D}^{2 *}$, Kang $\mathrm{Y}^{2}$, Dunn $\mathrm{K}^{3}$ and Kugel $\mathrm{G}^{4}$}

${ }^{1}$ BS, Tufts University School of Dental Medicine, USA

${ }^{2}$ Assistant Professor, Tufts University School of Dental Medicine, USA

${ }^{3}$ Assistant Director of Predoctoral Research, Tufts University School of Dental Medicine, USA

${ }^{4}$ Professor and Associate Dean for Research, Tufts University School of Dental Medicine, USA

*Corresponding author: Duong Tran, Assistant Professor, Tufts University School of Dental

Medicine, Boston, MA, 02111, USA, Tel: 6176363614; Fax: 6176366511; Email: Duong.tran@tufts.edu

\section{Abstract}

Introduction: This study aimed to compare ACTIVA ${ }^{\mathrm{TM}}$ BioACTIVE-Cement and two conventional orthodontic bracket cements regarding the demineralization resistance and shear bond strength (SBS).

Methods: Sixty extracted posterior teeth were randomly assigned to four groups: Transbond ${ }^{\mathrm{TM}}$ XT Adhesive Light Cure (Trans); GC Fuji ORTHO ${ }^{\mathrm{TM}}$ (Fuji); ACTIVA ${ }^{\mathrm{TM}}$ BioACTIVE-Cement without acid-etching (ActivaWOE); ACTIVA ${ }^{\mathrm{TM}}$ BioACTIVE-Cement with acid-etching (ActivaWE). Prior to bonding, all teeth were screened with DIAGNOdent ${ }^{\mathrm{TM}}$ to determine no demineralization was present and were assessed with MicroMet 2104 for Vickers microhardness at 12 standardized points on enamel. Brackets were bonded to the buccal surface of each tooth according to manufacturers' instructions. All samples were placed in a 0.05 $\mathrm{M}$ acetate buffer, pH 5.0 for 96 hours. After demineralization, brackets were de-bonded with a universal testing machine to determine SBS. All samples were tested again with DIAGNOdent to detect demineralization, and with MicroMet for Vickers microhardness at points $50 \mu \mathrm{m}$ distal (occlusal and cervical) and $50 \mu \mathrm{m}$ cervical (mesial and distal) to each original standardized point. DIAGNOdent values, Vickers microhardness, and SBS between the four groups were compared.

Results: Microhardness reductions after demineralization were 56.4, 58.7, 100.1, and 126.9 for ActivaWOE, ActivaWE, Fuji, and Trans, respectively. The microhardness reductions of ActivaWOE and ActivaWE were not statistically different. SBS was 8.9 MPa for Trans, 7.6 MPa for ActivaWE, 3.8 MPa for Fuji, and 2 MPa for ActivaWOE. There was no significant difference in SBS between ActivaWE and Trans.

Conclusion: ActivaWE for 10 seconds provided a similar SBS while significantly reduced the demineralization of enamel around brackets in comparison with the well-established resin cement, Transbond ${ }^{\mathrm{TM}}$ XT Adhesive Light Cure.

Keywords: ACTIVA ${ }^{\mathrm{TM}}$ BioACTIVE-Cement; Orthodontic bracket cements; White Spot Lesions; Bracket Cementation

Abbreviations: SBS: Shear Bond Strength; WSLs: White Spot Lesions; CPP-ACP: Casein Phosphopeptides-Amorphous Calcium Phosphate; GIC: Glass-Ionomer Cements.

\section{Introduction}

Fixed orthodontic appliances make oral hygiene maintenance difficult, resulting incipient enamel caries with subsurface demineralization beneath an intact surface layer 


\section{Open Access Journal of Dental Sciences}

of enamel around the appliances [1]. The demineralization is caused by acid produced by cariogenic bacteria in dental plaque accumulated around the appliances, often presenting as white spot lesions (WSLs) or early caries lesions [2]. 28 - 73\% of orthodontic treated patients developed WSLs [35] with an average of 2.4 white spots per patients [4]. WSL may appear within one month of bracket placement, $[2,6]$ primarily in the gingival area of the buccal surfaces. WSLs influence patients' aesthetics and satisfaction with the treatment and often can be very difficult to be improved and completely resolved.

Various methods have been recommended for preventing WSLs such as oral hygiene control, fluoride products (fluoride toothpastes, fluoride varnishes, fluoride rinses, fluoride-containing bonding materials, fluoride-releasing cements), [7-11] casein phosphopeptides-amorphous calcium phosphate (CPP-ACP), [12] resin sealants, glass ionomer cements, antibacterial cements, bioactive cement, antibacterial rinses, and lasers [4]. The effectiveness of these methods varies by studies and no consensus has been made regarding the best method [13]. Weak evidence indicated that glass-ionomer cements (GIC) performed better than composite resins in preventing demineralization [14]. However, GIC has higher bond failures than adhesive strength, resulting in more bracket failures [15-17]. With shortcomings in each material, attempts have been made to look for a new material that is not only has high bond strength, but also has properties to reduce prevalence of WSLs.

Recently, bioactive materials gain an increasing interest from dental professionals [18]. Among them, ACTIVA BioACTIVE-Cement ${ }^{\mathrm{TM}}$ (Pulpdent) is able to continuously release and recharge calcium, phosphate and fluoride, stimulating mineral apatite crystal formation at the materialtooth interface [19]. It is an ionic resin cement containing a bioactive resin matrix, shock-absorbing resin component, and reactive ionomer glass fillers. These components actively exchange the ions with saliva and tooth structure. Therefore, the cement was showed to resist to demineralization, promote remineralization, reduce microleakage, and prevent caries [19].

These features make the ACTIVA BioACTIVE-Cement a good candidate for cementing brackets and bands. The manufacturer stated that ACTIVA BioACTIVE products combine the benefits of composites, glass ionomers and RMGIs without their disadvantages. If the ACTIVA BioACTIVECement can both reduce enamel demineralization and demonstrate adequate shear bond strength, it may significantly improve orthodontic care in many clinical applications. This study aimed to compare ACTIVA ${ }^{\mathrm{TM}}$ BioACTIVE-Cement (Pulpdent) with two conventional orthodontic bracket cements, Transbond ${ }^{\mathrm{TM}} 3 \mathrm{M}$ XT Lightcure Adhesive (composite-based) and GC Fuji ORTHO ${ }^{\mathrm{TM}}$ (glass ionomer) regarding the demineralization resistance and shear bond strength (SBS).

\section{Material and Methods}

\section{Specimen Preparation}

Sixty extracted posterior teeth were collected, cleaned and stored in a $10 \%$ bleach solution. Prior to bonding, all teeth were screened with DIAGNOdent ${ }^{\mathrm{TM}}(\mathrm{KaVO})$ to determine no demineralization was present. The DIAGNOdent measures laser fluorescence within the mineral structure of the tooth [20]. As the incident laser light is disseminated into the site, two-way hand piece optics allows the unit to simultaneously quantify the reflected laser light energy. A DIAGNOdent score of 7 or more indicated that demineralization has occurred, higher numbers yielding more demineralization [21]. Samples with a score in the non-demineralized range, between three and seven, were used [22]. Teeth surfaces were polished with pumice using prophy cup and rinsed thoroughly with water. The teeth were randomly assigned into four experimental groups: Transbond XT Adhesive Light Cure (3M Unitek) (Trans); GC Fuji ORTHO (GC America) (Fuji); ACTIVA BioACTIVE-Cement without acid-etching (Pulpdent) (ActivaWOE); ACTIVA BioACTIVE-Cement with acid-etching (Pulpdent)(ActivaWE).

\section{Bracket Cementation}

Orthodontic brackets (OrmcoTM) ( $3.83 \times 2.95 \times 2.48 \mathrm{~mm})$ were bonded to the buccal surface of each tooth according to manufacturers' instructions. For the Tran, Fuji, and ActivaWE groups, teeth's surfaces were acid-etched with UltraEtch 35\% Phosphoric acid (Ultradent) for 10 seconds respectively. For the ActivaWOE group, teeth's surfaces were not etched with a conditioner or etching gel before cementation. The brackets were placed onto the tooth's surface, adjusted to its final position, and pressed firmly onto the tooth. The tip of an explorer was used to gently remove any excess material prior to curing, making sure the bracket placement was not compromised. Each bracket was light cured (DEMI, Kerr) for 10 seconds for each side of the bracket (4 sides).

\section{Microhardness Assessment}

Vickers microhardness was measured before bracket cementation and after bracket debonding using the MicroMet 2104 (Buehler). The measurement was performed with a pyramid-shaped diamond indenter using a vertical load of 500 gf for 20 seconds. Before cementation, the microhardness was measured at 12 standardized points on enamel, located 100 micrometers adjacent to edge of the area where each bracket was placed (Figure 1), with three points on each 
edge (occlusal, mesial, distal, and cervical). After the bond strength and demineralization tests, the microhardness was measured again 50 micrometers distal (if located occlusal and cervical) and 50 micrometers cervical (if located mesial and distal) to each original standardized point (Figure 2). The 12 microhardness values measured per tooth were averaged for each tooth.

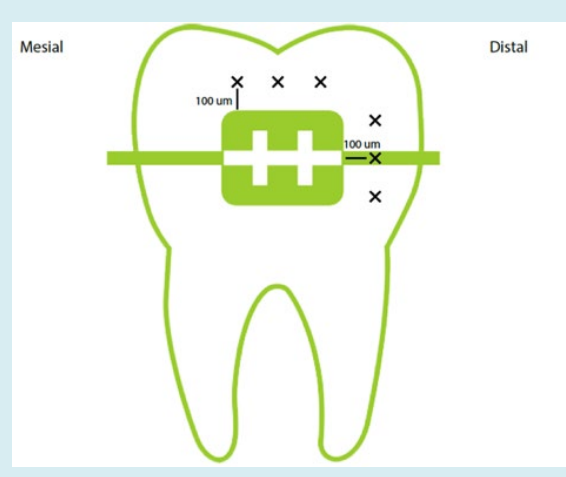

Figure 1: Baseline Vickers Microhardness.

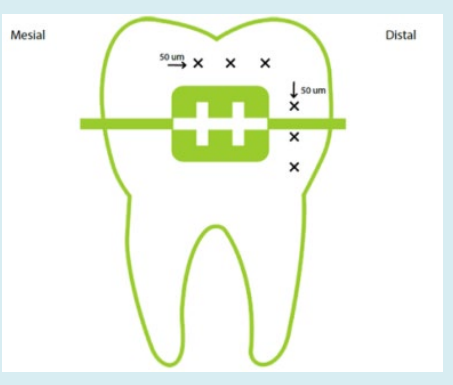

Figure 2: Post-Demineralization Vickers Microhardness.

\section{Teeth Demineralization}

After cementing brackets, the teeth were then mounted on acrylic blocks and immersed in a demineralization solution composed of $2 \mathrm{~mL}(0.05 \mathrm{M}$ acetate buffer, $\mathrm{pH}$ 5.0) [23]. Samples were immersed into the acidic solution for 96 hours, representing three months of real time [24]. After the 96 hour demineralization period, the teeth were removed from the solution. The DIAGNOdent was used to measure demineralization around each bracket (Figure 2).

\section{Shear Bond Strength}

After demineralization, acrylic blocks containing teeth with bonded brackets were stored in distilled water at $37^{\circ} \mathrm{C}$. Within one week of demineralization, the Instron $® 5566 \mathrm{~A}$ (Norwood, MA) model was used to test the shear bond strength (SBS) of each group with a $1 \mathrm{~K}$ load cell and crosshead speed of $1 \mathrm{~mm} /$ minute [25]. Acrylic blocks were mounted on a jig in a position that the bracket-tooth interfaces were parallel to the shear force created by the crosshead's metal rod moving down occluso-gingivally. The SBS was calculated by dividing the debonding force by the bracket base surface area and reported in megapascal (MPa).

\section{Sample Size}

The statistical software Stata 15 was used for sample size calculation. Published papers reported that the shear bond strengths were 23.6 \pm 9.2 MPa for ACTIVA BioACTIVE Cement and 15.5 $\pm 3.5 \mathrm{MPa}$ for Transbond 3M XT Light Cure $[25,26]$. To detect a mean difference of $8.1 \mathrm{MPa}$ in the shear bond strength between two groups, 30 teeth $(n=15$ per group) will achieve a power of $85 \%$ and significance level of 0.05 . The minimum sample size was determined to be 15 teeth per group for a total of 45 teeth.

\section{Data Analysis}

Descriptive statistics (means and standard deviations) were calculated for DIAGNOdent values, microhardness, and SBS. One-way ANOVA with Bonferroni correction for posthoc pairwise comparisons were used to determine difference in DIAGNOdent values, microhardness, and SBS between the four groups. Additionally, to account for the baseline difference in microhardness between groups, a multivariable linear regression was used with the post-demineralization microhardness as the dependent variable and the baseline microhardness and group as independent variables. All p-values less than 0.05 was considered statistically significant. The statistical software Stata (version 15) was used for the analysis.

\section{Results}

Baseline DIAGNOdent values were not significantly different between the four groups (Figure 3). After demineralization, the DIAGNOdent value for Trans was significantly higher compared to other groups. Baseline microhardness was significantly different between groups with the ActivaWE having the highest value (Figure 4, Table 1). The microhardness values post-demineralization decreased in all four groups (Figure 4). Microhardness reductions after demineralization were 56.4, 58.7, 100.1, and 126.9 for ActivaWOE, ActivaWE, Fuji, and Trans, respectively. The microhardness reductions of ActivaWOE and ActivaWE were not statistically different. SBS was $8.9 \mathrm{MPa}$ for Trans, 7.6 MPa for ActivaWE, 3.8 MPa for Fuji, and $2 \mathrm{MPa}$ for ActivaWOE. There was no significant difference in SBS between ActivaWE and Trans (Table 1). Multivariable linear regression analysis showed that the post-demineralization microhardness of ActivaWOE, Fuji, and ActivaWE were 49.9, 3.4 , and 59.6 points higher in average compared to Trans, respectively, after adjusting for the difference in the baseline 
microhardnesss between the 4 groups (Table 2).

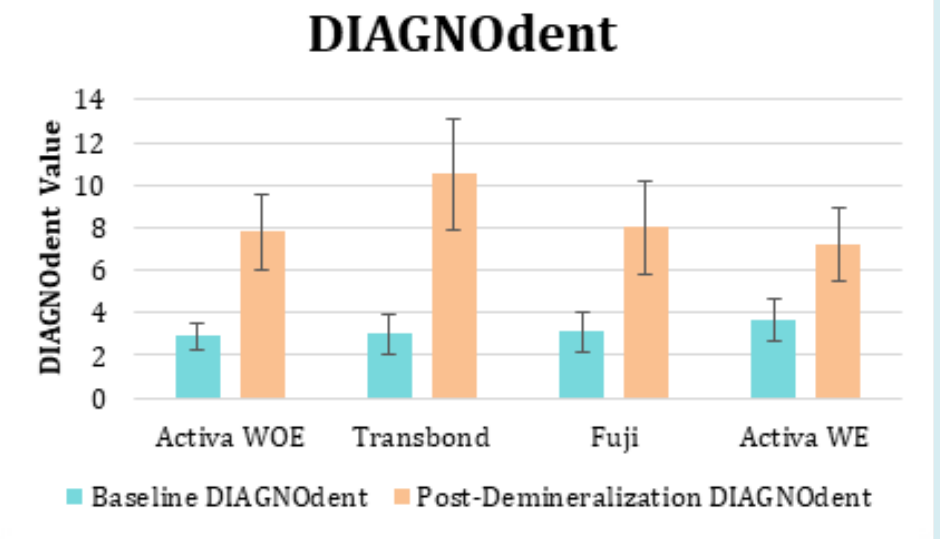

Figure 3: DIAGNOdent values at baseline and post-demineralization.

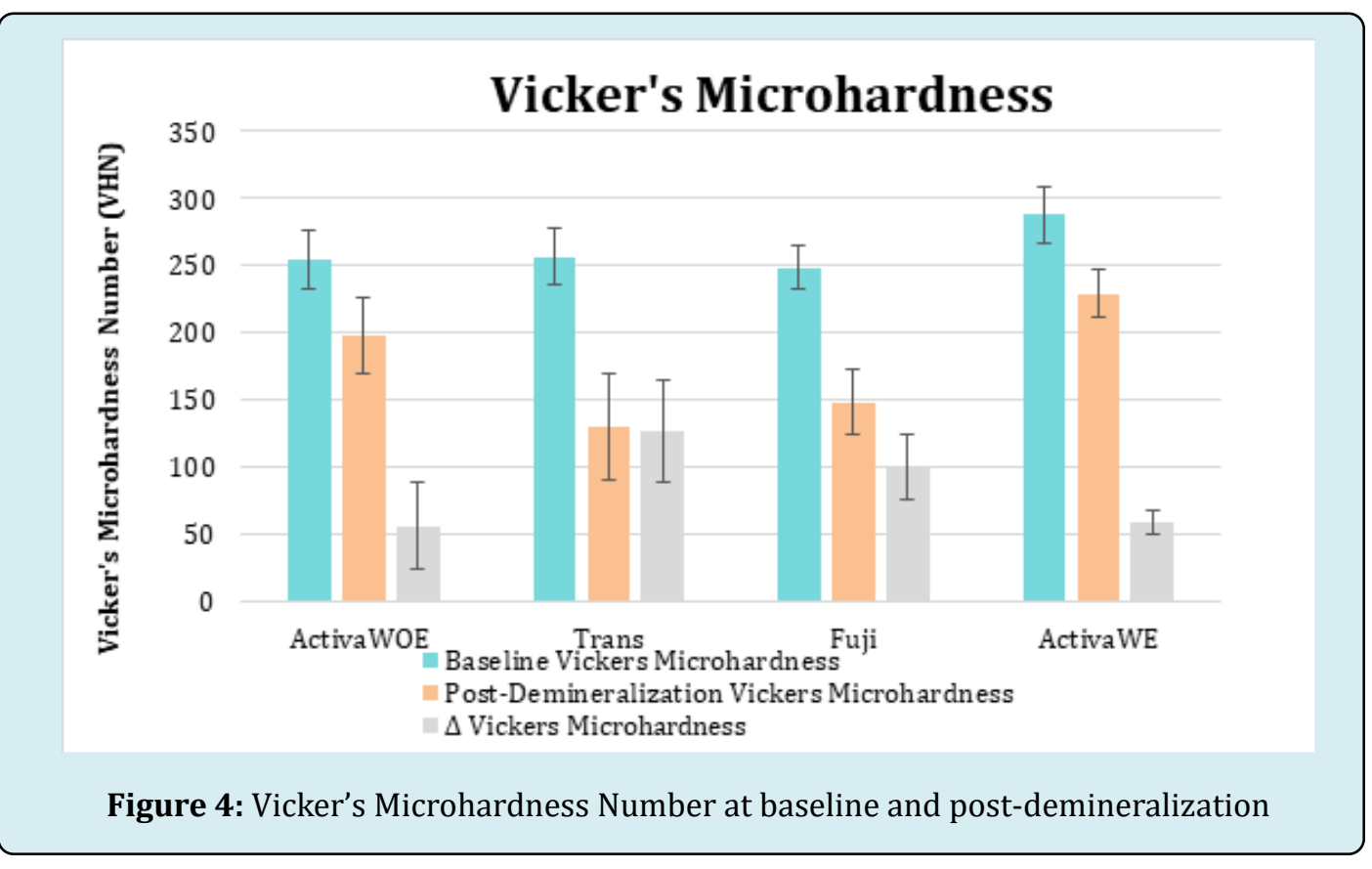

\begin{tabular}{|c|c|c|c|c|}
\hline GROUP & $\begin{array}{c}\text { Baseline Vickers } \\
\text { Microhardness }\end{array}$ & $\begin{array}{c}\text { Post-Demineralization } \\
\text { Vickers Microhardness }\end{array}$ & $\begin{array}{c}\Delta \text { Vickers } \\
\text { Microhardness }\end{array}$ & SBS \\
\hline ActivaWOE & $254.2 \pm 21.7^{\mathrm{a}}$ & $197.8 \pm 28.6^{\mathrm{a}, \mathrm{b}, \mathrm{c}}$ & $56.4 \pm 31.8^{\mathrm{a}, \mathrm{b}}$ & $2.0 \pm 1.2^{\mathrm{a}, \mathrm{b}}$ \\
\hline Trans & $257.0 \pm 21.6^{\mathrm{b}}$ & $130.1 \pm 39.8^{\mathrm{a}, \mathrm{d}}$ & $126.9 \pm 37.4^{\mathrm{a}, \mathrm{c}}$ & $8.9 \pm 5.7^{\mathrm{a}, \mathrm{c}}$ \\
\hline Fuji & $248.3 \pm 16.2^{\mathrm{c}}$ & $148.3 \pm 24.2^{\mathrm{b}, \mathrm{c}}$ & $100.1 \pm 24.3^{\mathrm{b}, \mathrm{d}}$ & $3.8 \pm 2.0^{\mathrm{c}}$ \\
\hline ActivaWE & $288.1 \pm 21.4^{\mathrm{a}, \mathrm{b}, \mathrm{c}}$ & $229.4 \pm 17.2^{\mathrm{c}, \mathrm{d}, \mathrm{e}}$ & $58.7 \pm 8.7^{\mathrm{c}, \mathrm{d}}$ & $7.6 \pm 4.8^{\mathrm{b}}$ \\
\hline ANOVA p value & $<0.01$ & $<0.001$ & $<0.001$ & $<0.001$ \\
\hline
\end{tabular}

In each column, mean microhardness or SBS values of two groups with the same lowercase letter were significantly different. $\Delta=$ Baseline Vickers Microhardness - Post-Demineralization Vickers Microhardness.

Table 1: Vickers Microhardness and Shear Bond Strength Values. 
Open Access Journal of Dental Sciences

\begin{tabular}{|c|c|c|c|}
\hline Variables & Regression coefficients & $\mathbf{9 5 \%}$ CI & p \\
\hline Group & & & $<0.001$ \\
\hline ActivaWOE & 69.3 & $49.9-88.7$ & 0.02 \\
\hline Trans & reference & reference & $<0.001$ \\
\hline Fuji & 23.1 & $3.4-42.7$ & 0.002 \\
\hline ActivaWE & 81.8 & $59.6-104$ & \\
\hline Baseline microhardness & 0.6 & $02-0.9$ & \\
\hline Total adjusted R $\mathrm{R}^{2}$ & 0.7 & & \\
\hline
\end{tabular}

Post-demineralization microhardness was adjusted for baseline microhardness difference between groups. The reference category was Transbond XT Adhesive Light Cure.

Table 2: Multivariable linear regression analysis for comparing post-demineralization microhardness between groups.

\section{Discussion}

This in-vitro study presented promising properties of the bioactive material, ACTIVA BioACTIVE-Cement with acid-etching, in comparison with other materials for cementing orthodontic brackets. ACTIVA BioACTIVE-Cement significantly reduced the demineralization of enamel around brackets while providing a similar resistance to withstand the forces applied during the orthodontic treatment in comparison with the well-established resin cement, Transbond XT Adhesive Light Cure. Since the main usage of the ACTIVA BioACTIVE-Cement is crown cementation according to the manufacturer guideline, the findings of this study indicated a possibility of an additional use of the material for cementing orthodontic brackets.

The average baseline enamel Vickers microhardness of all samples in our study was $261.9 \pm 25.2$ which is similar to values reported in literature ranging from 270 to 360 for enamel [2,12,27-29]. Microhardness and of all samples decreased and DIAGNOdent values increased variously after acid challenge, indicating a demineralization of enamel. ACTIVA BioACTIVE-Cement provided the least enamel demineralization around the brackets, followed by GC Fuji ORTHO and Transbond XT Adhesive Light Cure. This finding corroborates with the literature reporting that using calcium, phosphate and fluoride-releasing dental materials during or after bonding orthodontic brackets successfully reduce demineralization of enamel around the brackets invitro and in vivo $[1,30,31]$. In addition, studies showed that glass ionomer cements suffered less demineralization on enamel and dentin/restoration interfaces when compared to composite resins [32].

ACTIVA BioACTIVE-Cement reduced enamel demineralization around the brackets due to its ability to release and recharge more calcium, phosphate and fluoride than glass ionomer and traditional RMGI Cements. It is composed of a bioactive ionic resin matrix, a rubberized resin component, and reactive ionomer glass fillers. These components allow the material to mimic the physical and chemical properties of the natural tooth. The oral environment has a fluctuating $\mathrm{pH}$, and ACTIVA responds through the release of calcium, phosphate, and fluoride. ACTIVA Cement, when placed in a phosphate buffered saline solution, was shown to have an increased concentration of calcium and phosphorus ions at the surface indicating the formation of mineral apatite deposits [33] and this layer of apatite integrates the cement with dentin [34]. ACTIVA was shown to have greater fluoride release compared to two commonly used glass ionomer and resin-modified glass ionomers [35]. Phosphate release contributes to apatite formation, and its release is $\mathrm{pH}$ dependent. At lower $\mathrm{pH}$ values ACTIVA releases phosphate, and this phosphate can interact with calcium and fluoride ions during periods of higher $\mathrm{pH}$ [36]. Ion release and apatite deposition are properties of ACTIVA that allow this material to have the potential to produce long-lasting and effective cementation.

There has not been much data about the shear bond strength of ACTIVA BioACTIVE-Cement. The bond strength of the orthodontic bracket must be able to withstand the forces applied during the orthodontic treatment. Reynolds [37] stated that 5.9-7.8 MPa resistances are sufficient to withstand masticatory forces. In our study, ACTIVA BioACTIVE-Cement with enamel etching and Transbond XT Adhesive Light Cure provided SBS values within this range. Additionally, our study showed that etching enamel prior to the bracket cementation with ACTIVA BioACTIVECement significantly increased SBS to a similar value of a well-established self-adhesive resin cement, Transbond XT Adhesive Light Cure. Claussen, et al. reported that ACTIVA BioACTIVE-cement and self-adhesive resin cement, RelyX ${ }^{T M}$ Unicem 2, showed no significantly different SBS on enamel [26]. Another study was conducted to compare the SBS of various cements when bonded to zirconia, e.max crowns, titanium, stainless steel, and dentin. The results showed that ACTIVA BioACTIVE Cement had statistically superior shear bond strength when compared to Rely $\mathrm{X}^{\mathrm{TM}}$ Unicem 2 Automix, Ceramir ${ }^{\circledR}$ Crown \& Bridge, and FujiCEM ${ }^{\mathrm{TM}} 2$ Cement [38]. According to the Pulpdent's instruction for use, the ACTIVA 
BioACTIVE-Cement is dedicated for crown and bridge cementation [39]. Our study's results corroborated with the literature supporting the potential use of the product for cementing orthodontic bracket.

There were several limitations in our study. First, although teeth were randomly assigned to groups, baseline microhardness was different between the groups. To account for the baseline difference, we also calculated and compared the change in microhardness after demineralization between groups and used linear regression model to adjust for the difference in the baseline difference. Second, we assessed the performance of the materials after the acid challenge only. This may not mimic the real situation of alternative demineralization and remineralization process in the oral cavity. We expect that ACTIVA BioACTIVE Cement may even perform better in clinical situation due to its bioactive characteristic. Third, curved surfaces of the teeth may affect the accuracy of microhardness. However, because we want to assess the surface microhardness around the bracket, this limitation is unavoidable. To reduce potential measurement errors, we measured microhardness at many points around the brackets and averaged them.

The findings of this study suggested an additional use of ACTIVA BioACTIVE Cement for cementing orthodontic brackets as well as future research. In this study, only one etching time of 10 seconds was used for etching enamel before applying ACTIVA BioACTIVE Cement. In order to develop a clinical instruction for using the material, different etching times should also be assessed for the optimal performance of the ACTIVA BioACTIVE Cement. Additionally, assessing the performance of the ACTIVA BioACTIVE Cement after demineralization was the first step, further study is needed to assess the effect of alternative demineralization and remineralization process both in vitro and in-vivo on the performance of ACTIVA BioACTIVE Cement.

\section{Conclusion}

The results of this study indicate that ActivaWE for 10 seconds has increased SBS while exhibiting less demineralization of tooth structure. This material may offer better strength of enamel clinically, while reducing the incidence of white spot lesions.

\section{References}

1. Khoroushi M, Kachuie M (2017) Prevention and Treatment of White Spot Lesions in Orthodontic Patients. Contem Clin Dent 8(1): 11-19.

2. Gorton J, Featherstone JD (2003) In vivo inhibition of demineralization around orthodontic brackets. Am J Ortho Dentofacial Orthop 123(1): 10-14.
3. Richter AE, Arruda AO, Peters MC, Sohn W (2011) Incidence of caries lesions among patients treated with comprehensive orthodontics. Am J Ortho Dentofacial Orthop 139(5): 657-664.

4. Brown MD, Campbell PM, Schneiderman ED, Buschang PH (2016) A practice-based evaluation of the prevalence and predisposing etiology of white spot lesions. The Angle Orthodontist 86(2): 181-186.

5. Sundararaj D, Venkatachalapathy S, Tandon A, Pereira A (2015) Critical evaluation of incidence and prevalence of white spot lesions during fixed orthodontic appliance treatment: A meta-analysis. J Int Soc Prev Community Dent 5(6): 433-439.

6. Lucchese A, Gherlone E (2013) Prevalence of white-spot lesions before and during orthodontic treatment with fixed appliances. Eur J Orthodont 35(5): 664-668.

7. Lovrov S, Hertrich K, Hirschfelder U (2007) Enamel Demineralization during Fixed Orthodontic TreatmentIncidence and Correlation to Various Oral-hygiene Parameters. Journal of Orofacial Orthopedics 68(5): 353-363.

8. Geiger AM, Gorelick L, Gwinnett AJ, Benson BJ (1992) Reducing white spot lesions in orthodontic populations with fluoride rinsing. AM J Orthod Dentofacial Orthop 101(5): 403-407.

9. Stecksen Blicks C, Renfors G, Oscarson ND, Bergstrand F, Twetman S (2007) Caries-preventive effectiveness of a fluoride varnish: a randomized controlled trial in adolescents with fixed orthodontic appliances. Caries Res 41(6): 455-459.

10. Vivaldi-Rodrigues G, Demito CF, Bowman SJ, Ramos AL (2006) The effectiveness of a fluoride varnish in preventing the development of white spot lesions. World J Orthod 7(2): 138-144.

11. Tanna N, Kao E, Gladwin M, Ngan PW (2009) Effects of sealant and self-etching primer on enamel decalcification. Part I: an in-vitro study. Am J Orthod Dentofacial Orthop 135(2): 199-205.

12. Behnan SM, Arruda AO, Gonzalez-Cabezas C, Sohn W, Peters MC (2010) In-vitro evaluation of various treatments to prevent demineralization next to orthodontic brackets. Am J Orthod Dentofacial Orthop 138(6): 711-717.

13. Mousavinasab SM, Meyers I (2009) Fluoride release by glass ionomer cements, compomer and giomer. Dent Res J 6(2): 75-81.

14. Rogers S, Chadwick B, Treasure E (2010) Fluoridecontaining orthodontic adhesives and decalcification in patients with fixed appliances: a systematic review. Am J Orthod Dentofacial Orthop 38(4): 390-398. 
15. Mandall NA, Millett DT, Mattick CR, Hickman J, Worthington HV, et al. (2002) Orthodontic adhesives: a systematic review. J Ortho 29(3): 205-210.

16. Turner PJ (1993) The clinical evaluation of a fluoridecontaining orthodontic bonding material. Br J Orthod 20(4): 307-313.

17. Sidhu SK, Nicholson JW (2016) A Review of GlassIonomer Cements for Clinical Dentistry. J Funct Biomater $7(3)$.

18. Manchir M (2018) ACE Panel report focuses on bioactive materials.

19. Pulpdent. ACTIVA BioACTIVE. A closer look at BioACTIVE Materials.

20. Bahrololoomi Z, Musavi SA, Kabudan M (2013) In vitro evaluation of the efficacy of laser fluorescence (DIAGNOdent) to detect demineralization and remineralization of smooth enamel lesions. J Conserv Dent 16(4): 362-366.

21. Shukla C, Maurya RK, Singh V, Tijare M (2016) Evaluation of changes in Streptococcus mutans colonies in microflora of the Indian population with fixed orthodontics appliances. Dent Res J 13(4): 309-314.

22. KaVo Dental KaVo DIAGNOdent Caries Detection Made Simple.

23. Nalbantgil D, Oztoprak MO, Cakan DG, Bozkurt K, Arun T (2013) Prevention of demineralization around orthodontic brackets using two different fluoride varnishes. Eur J Dent 7(1): 41-47.

24. Sudjalim TR, Woods MG, Manton DJ, Reynolds EC (2007) Prevention of demineralization around orthodontic brackets in vitro. Am J Orthod Dentofacial Orthop 131(6): 701-709.

25. Hellak A, Ebeling J, Schauseil M, Stein S, Roggendorf M, et al. (2016) Shear Bond Strength of Three Orthodontic Bonding Systems on Enamel and Restorative Materials. BioMed Res Int. 2016: 6307107.

26. Claussen K, Ludsteck M, Hader S, Hecht R (2017) Ion Release and Shear Bond Strength of Activa- Bioactive Cement and RelyX Unicem 2 IADR/AADR/CADR General Session \& Exhibition; San Francisco.

27. Lopatiene K, Borisovaite M, Lapenaite E (2016) Prevention and Treatment of White Spot Lesions During and After Treatment with Fixed Orthodontic Appliances: a Systematic Literature Review. J Oral Maxillofac Res $7(2): 1$.

28. Kumar Jena A, Pal Singh S, Kumar Utreja A (2015) Efficacy of resin-modified glass ionomer cement varnish in the prevention of white spot lesions during comprehensive orthodontic treatment: a split-mouth study. J Orthod 42(3): 200-207.

29. Fallahinejad Ghajari M, Eslamian L, Naji Rad A, Morovati SP (2015) Efficacy of Glass Ionomer Cements for Prevention of White Spot Lesions During Orthodontic Banding: A Randomized Clinical Trial. J Dent (Tehran, Iran) 12(12): 913-920.

30. Kau CH, Wang J, Palombini A, Abou-Kheir N, Christou T (2019) Effect of fluoride dentifrices on white spot lesions during orthodontic treatment: A randomized trial. Angle Orthod 89(3): 365-371.

31. Oz AZ, Oz AA, Yazicioglu S (2017) In vivo effect of antibacterial and fluoride-releasing adhesives on enamel demineralization around brackets: A micro-CT study. Angle Orthod 87(6): 841-846.

32. Seixas LC, Seixas FH, Ciccone JC, Souza WCS, Palma-Dibb RG (2004) Demineralization around restorations with different restorative materials containing fluoride. Mate Res 7(2): 235-240.

33. Chao W, Perry R, Kugel G (2016) Surface Deposition Analysis of Bioactive Restorative Material and Cement. J Dent Res 95: 1313.

34. Garcia-Godoy F, Morrow B (2016) Profilometry bioactive dental materials analysis and evaluation of dentin integration. J Dent Res 95: 1828.

35. Slowikowski L (2014) Fluoride ion release and recharge over time in three restoratives. J Dent Res 93: 268.

36. Pulpdent $\mathrm{pH}$ dependence on the phosphate release of Activa ionic materials.

37. Reynolds I (1975) A review of direct orthodontic bonding. Br J Orthod 2(3): 171-178.

38. Afutu R, Daddona J, Dunn K, Finkelman M, Tran A, et al. (2019) Shear Bond Strength of Several Dental Cements. Open Access Journal of Dental Sciences 4(4): 1-5.

39. Pulpdent. ACTIVA ${ }^{\mathrm{TM}}$ BioACTIVE-CEMENT ${ }^{\mathrm{TM}}$. 